\section{THU0318 CORONARY ARTERY DISEASE IN PATIENTS WITH BEHÇET'S DISEASE: A RETROSPECTIVE, SINGLE CENTER STUDY}

H. Chen ${ }^{1}$, Y. Zhang ${ }^{2}$, W. Chen ${ }^{3}$, J. Liu ${ }^{1}$, L. Wang ${ }^{1}$, F. Zhang ${ }^{1}$, W. Zheng ${ }^{1}$. ${ }^{1}$ Rheumatology, Peking Union Medical College Hospital, BEIJING; ${ }^{2}$ Oncology and immunology, The fourth hospital of Hebei Medical University, Hebei; ${ }^{3}$ Cardiology, Peking Union Medical College Hospital, BEIJING, China

Background: Behçet's disease (BD) is a chronic inflammatory disease affecting various size of arteries and veins. Coronary artery disease (CAD), a lifethreatening condition, is rarely reported in patients BD.

Objectives: To investigate the clinical characteristics of BD patients complicated with $C A D$, and to elucidate the potential risk factors of CAD in BD patients.

Methods: We retrospectively reviewed all the medical records of $B D$ patients who were admitted to our institute from 2001 to 2016. CAD was defined as aneurysm, stenosis and (or) occlusion of coronary arteries confirmed by angiography or contrast-enhanced computer tomography. BD patients with CAD and age- and gender-matched BD patients without CAD (at 1:3 ratio) were enrolled. Demographic, clinical and laboratory data were systemically collected, analyzed and compared between two groups.

Results: In total, 19 patients, including 17 male and 2 female, were complicated with CAD. The mean onset age of $\mathrm{BD}$ was 34 and the mean duration from the onset of $\mathrm{BD}$ to the diagnosis of $\mathrm{CAD}$ was 4.1 year. Angina pectoris $(8 / 19)$ and acute myocardial infarction (8/19) were the most common cardiac symptoms, arrhythmia was presented in one patient, and three patient remained asymptomatic. Coronary artery aneurysm, stenosis and occlusion were presented in 9,13 and 3 patients, respectively. Smoking $(7 / 19)$ was frequently observed, while hypertension (3/16), diabetes mellitus (2/19), obesity (1/19) and alcohol consumption (1/19) were rarely present. Additionally, seven arterial and two venous extra-cardiac vasculopathies were presented. Oral ulceration (19/19) and skin lesions (16/19) were the most common BD-associated symptoms. Comparing with $\mathrm{BD}$ patients without $\mathrm{CAD}$, patients with $\mathrm{BD}$ presented with higher ESR (mean, 34.4 vs $16.3 \mathrm{~mm} / \mathrm{hr}, \mathrm{p}=0.0018$ ) and CRP (mean, $36.4 \mathrm{vs} 12.2 \mathrm{mg} / \mathrm{L}$, $p=0.002$ ), more frequency of skin lesions ( $84 \%$ vs $55 \%, p=0.0334$ ) and pathergy reactions $(37 \%$ vs $26 \%, p=0.0103)$. Furthermore, multivariate analysis confirmed that elevated CRP was a independent risk factor of CAD $(\mathrm{OR} 1.032,95 \% \mathrm{Cl}$ $1.011-1.053, p=0.003)$

Conclusions: $C A D$, a rare complication of $B D$, predominately affect male patients. $\mathrm{BD}$ patients with $\mathrm{CAD}$ presented with active $\mathrm{BD}$ disease symptoms and elevated inflammatory markers, which implicated aberrant vascular inflammation was the key mechanism of CAD in BD patients. CRP, but not traditional CAD risk factors, was the risk factor of $C A D$ in $B D$ patients.

Disclosure of Interest: None declared

DOI: 10.1136/annrheumdis-2017-eular.5991

\section{THU0319 MR ANGIOGRAPHY FOR EVALUATION OF VASCULAR INFLAMMATION IN ELDER PATIENTS WITH LARGE VESSEL VASCULITIS}

I. Smitienko ${ }^{1}$, P. Novikov ${ }^{2}$, E. Atyasova ${ }^{1} .{ }^{1}$ Medical Center "Petrovskie Vorota"; ${ }^{2}$ Sechenov First Moscow State Medical University, Moscow, Russian Federation

Objectives: The aim of the study was to evaluate the feasibility of MR angiography (MRA) for evaluation of vascular inflammation in elder patients with large vessel vasculitis.

Methods: 16 patients with established on PET with 18F-FDG large vessel vasculitis (14 female and 2 male; average age 66 years) were enrolled in our study. 14 patients had got giant cell arteritis (just only 3 biopsy-proven cases), 2 patients with isolated aortitis of thorax aorta and 2 cases of PMR-associated arteritis. The average duration of any disease was 8 months. All patients underwent MRA with or without contrast enhancement of aorta and its branches at 1.5 Tesla (Siemens Magnetom Essenza). All patients had repeated MRA at 6 and 12 months. All images were studied by one specialist. We evaluated the role of mural oedema as a sign of activity of vasculitis. The results of MR-angiography were compared with clinical and laboratory data, ultrasound and PET with 18F-FDG

Results: A total of 42 MRA were obtained in 16 patients. Significant mural oedema of thorax aorta or large arteries was shown by imaging in 20 of 42 cases $(48.0 \%)$ and correlated with clinical and laboratory signs of vasculitis activity, progressive arterial stenosis detected by ultrasound and increased uptake of 18F-FDG on PET. 12 patients with high degree of inflammation on MRA were streroid-naïve. In 22 cases (52\%), MRA had showed mild or moderate oedema of the arteries' wall. Low-moderate activity of vascular inflammation in our patients was associated with moderate or high of immunosuppressive therapy (prednisolone 40-60 mg/day). However, gradual reduction in the intensity of immunosuppression in 4 patients with mild mural oedema was associated with development of relapse of large vessel vasculitis. Notably, contrast enhancement did not improve significantly edema imaging.

Conclusions: Visualisation of artery wall oedema by MRA may be a usefull approach to detect persisting inflammation in elder patients with large vessel vasculitis.

Disclosure of Interest: None declared

DOI: 10.1136/annrheumdis-2017-eular.3616

\section{THU0320 DIFFERENT PATTERNS OF VASCULAR INVOLVEMENT IN PET/CT ACCORDING TO CRANIAL SYMPTOMS IN BIOPSY PROVEN GIANT CELL ARTERITIS, A PRELIMINARY STUDY}

J. Mestre Torres ${ }^{1}$, I. Navales ${ }^{2}$, F. Martínez ${ }^{1}$, J. Loureiro ${ }^{1}$, S. Bujan ${ }^{1}$, C. Pérez ${ }^{1}$, M. Simó ${ }^{2}$, R. Solans ${ }^{1} .{ }^{1}$ Internal Medicine; ${ }^{2}$ Nuclear Medicine, Hospital Vall d'Hebron, Barcelona, Spain

Background: GCA is the most frequent vasculitis in patients over 50 years old. It involves large vessels (LV) such as aorta, carotid, vertebral and subclavian arteries. After the development of new imaging techniques, extracranial LV involvement has been increasingly described. Past studies showed a trend in worse prognosis in those patients with aortitis at diagnosis but larger studies are needed to determine the clinical implications of these results. Correlation between symptoms at diagnosis and PET evaluation has not been done.

Objectives: To evaluate aortic and supraaortic involvement in a new onset GCA cohort through PET/CT. To compare aortic and supraaortic involvement in patients with and without cranial ischemic symptoms.

Methods: Prospective study including all newly diagnosed biopsy-proven GCA patients in 2016 according to ACR criteria. PET/CT was performed during initial evaluation. Clinical data, blood tests and PET/CT results were recorded. Ischemic symptoms included jaw claudication $(\mathrm{JC})$ and visual symptoms. Analysis was made using Stata IC/14. Patients were compared according to clinical presentation (presence vs absence of ischemic symptoms) with Fisher exact test.

Results: 18 patients were included (63.6\% were women). At diagnosis age was $77.4 \pm 8.7$ years (mean \pm SD). Patients presented headache $(78.6 \%)$, polymyalgia rheumatica (14.3\%), constitutional syndrome $(42.8 \%)$, JC (42.9\%) and visual symptoms (35.7\%). On physical examination pulse was decreased in $78.6 \%$ of patients. Blood tests showed anemia in $71.4 \%$ with an ESR $90.41 \pm 33 \mathrm{~mm} / \mathrm{h}$ (mean $\pm \mathrm{SD}$ ). PET/CT was performed after (median, intercuartilic range) 6.5 days (2 - 10 days). Analysis of vascular uptake was semiquantitative. Aortic involvement was present in $42.9 \%$ of patients. Supraaortic and vertebral arteries were involved in $42.8 \%$. Baseline characteristics were similar to other cohorts. At the time of diagnosis, proportion of aortitis was significantly lower $(p=0.049)$ in patients with ischemic symptoms when compared to those without. We could not find any differences between these two groups when analyzing the presence of increased vascular uptake in carotid and vertebral arteries. Limitations of the study are the small size of the cohort and the variability of the PET/CT protocols among literature. However, we think this study is important because it shows the differences between these two subgroups of GCA.

Conclusions: In this study, patients with cranial ischemic symptoms showed less involvement of large vessels when compared to those GCA patients that presented with systemic symptoms. These results suggest that there may be different physiopathological subsets leading to GCA. Further studies are needed to better understand these mechanisms.

Disclosure of Interest: None declared

DOI: 10.1136/annrheumdis-2017-eular.3528

\section{THU0321 ALTERATIONS IN THE PERIPHERAL B CELL COMPARTMENT IN PATIENTS WITH EOSINOPHILIC GRANULOMATOSIS WITH POLYANGIITIS (CHURG-STRAUSS SYNDROME)}

M. Rizzi, M. Engesser, R.E. Voll, A. Troilo, I. Janowska, N. Venhoff, J. Thiel. Rheumatology/Clinical Immunology, University Hospital Freiburg, Freiburg, Germany

Background: Eosinophilic granulomatosis with polyangiitis (EGPA) belongs to the group of ANCA associated vasculitides. While the combination of asthmatic symptoms and vasculitis characterize the disease clinically, eosinophilia and increased serum IgE concentrations are serologic hallmarks. The role of $B$ lymphocytes in EGPA has not been defined so far, but therapeutic response to rituximab in EGPA points towards a role of B-cells in the pathogenesis of EGPA. Objectives: To characterize the peripheral B cell compartment in patients with EGPA, to analyze the in vivo potential of $B$ lymphocytes to class-switch to $\lg E$, and to assess in vitro the differentiation of naïve $B$ cells to lgE-secreting plasmablasts. Methods: Laboratory work-up included ANCA-status, eosinophils, $\lg E, \lg G, \lg$ A, $\operatorname{lgM}$, and peripheral $\mathrm{CD} 19^{+} \mathrm{B}$-cell count. B cell subpopulations (naïve, marginal zone, class-switched $\mathrm{B}$ cells and plasmablasts) were analyzed by staining PBMCs with fluorescent-labeled monoclonal antibodies against: CD27, CD20, CD38, IgD, $\lg G$, IgA, IgE, CD21, and BAFF-R. For in vitro differentiation assays magnetically isolated $B$ lymphocytes from EGPA patients and aged matched healthy controls were stimulated with CD40L and IL-21 and IL-4 in enriched Iscoves' medium supplemented with $10 \% \mathrm{FCS}, 1 \mu \mathrm{g} / \mathrm{mL}$ insulin, $2.5 \mu \mathrm{g} / \mathrm{mL}$ apo-transferrin, $1 \%$ nonessential amino acids, $2 \mathrm{mmol} / \mathrm{L}$ glutamine, and $1 \mu \mathrm{g} / \mathrm{mL}$ reduced glutathione. Starting the culture with equal number of $B$ cells, the absolute number of plasmablasts, and IgE class switched cells after 9 days was determined by counting the events in the $\mathrm{CD} 27^{\text {high }} \mathrm{CD} 38^{\text {high }}$ gate or the $\lg \mathrm{G} / \mathrm{A} / \mathrm{D} \lg \mathrm{E}^{+}$gate by flow cytometry. IgE secretion in the supernatant was measured by ELISA.

Results: 18 patients (8 females, median age 59 years) with EGPA diagnosed according to ACR and CHC-criteria were included into the study. $22 \%$ of patients were ANCA-positive. Immunosuppressive therapy was azathioprine in 11 patients, methotrexate in 3 patients, and leflunomid or mycophenolate in one patient each and two patients received no immunosuppressive treatment. 7 patients had a history of a prior cyclophosphamide therapy. Median lymphocyte count was 
normal $\left(1.2 \times 10^{3} / \mu \mathrm{l}\right.$; normal range 1.1 to $3.2 \times 10^{3} / \mu$ I) but peripheral $\mathrm{B}$ cell numbers were markedly diminished $(37 / \mu \mathrm{l}$, normal range: $100-500 / \mu \mathrm{l})$. While the percentage of naĩve B cells $(53 \%)$ and marginal zone like B cells $(10.5 \%)$ were within normal range, the percentage of class-switched memory B cells was high $(28.2 \%)$. To assess the in vitro class switch capacity to $\lg E$ the number of IgE class-switched cells after 9 days of culture was determined by counting the events in the $\lg \mathrm{G} / \mathrm{A} / \mathrm{D}-\mathrm{lgE}+$ gate. The percentage of $\mathrm{lgE}$-switched cells was $6.8 \%$ (range: $0.3 \%>34 \%$ ) and not statistically different from healthy controls.

Conclusions: In the EGPA-patients we report we observed markedly diminished B-cells despite of normal lymphocyte counts. Within the B cell compartment, there was a shift towards later $B$ cell maturation stages. The in vitro B-cell development to IgE class-switched cells was not increased in EGPA-patients pointing towards a non-B cell intrinsic mechanism.

Disclosure of Interest: None declared

DOI: 10.1136/annrheumdis-2017-eular.6507

\section{THU0322 DESCRIPTIVE STUDY OF ASIAN INDIAN PATIENTS WITH RHEUMATOID VASCULITIS IN RETROSPECT: A SINGLE, TERTIARY CARE CENTRE EXPERIENCE}

J. Mathew ${ }^{1}$, A. Ganapati ${ }^{1}$, R. Goel ${ }^{1}$, S. Pulikool ${ }^{1}$, A.J. Mathew ${ }^{1}$, R. Janardhana ${ }^{1}$, M. Gowri ${ }^{2}$, D. Danda ${ }^{1} .{ }^{1}$ Rheumatology; ${ }^{2}$ Biostatistics, Christian Medical College, Vellore India, Vellore, India

Background: Rheumatoid vasculitis (RV) is a severe extra-articular manifestation of rheumatoid arthritis (RA), with high morbidity and mortality reported in literature Objectives: To describe the Asian Indian perspective on RV patients, their clinico-laboratory features and their outcome along with the factors affecting them Methods: A retrospective review of electronic medical records of 8984 RA patients from January 2007 to August 2016, was done for those satisfying Scott \& Bacon criteria for RV ${ }^{1}$. Probable RV was defined as patients not satisfying Scott \& Bacon Criteria, but were managed like RV after exclusion of alternate diagnosis. Birmingham Vasculitis activity score (BVAS) version $3^{2}$ was used for monitoring activity of RV

Results: 63 patients of RV were identified, with a study period prevalence of $0.7 \%$, in our RA cohort. $33(52.4 \%)$ patients were female. Mean age of patients was $50.7 \pm 11.5$ years with median duration of RA being 6 years. Involvement of Peripheral Nervous System (PNS) was the commonest manifestation of RV in 52/63 (82.5\%) patients followed by skin in 34/63 (53.9\%) patients. Rheumatoid Nodule was seen in $14 / 63(22.2 \%)$ patients. Percentage of current and ex-smokers combined,was same as rheumatoid nodule prevalence. $52(82.5 \%)$ patients had biopsy evidence of vasculitis.26/51 (50.9\%)patients were started on mycophenolate mofetil, $13 / 51(25.5 \%)$ patients on cyclophosphamide, $8 / 51$ (15.7\%) patients on azathioprine, $4 / 51(7.8 \%)$ patients on Methotrexate as immunosuppressive (IS) agent along with mean dose of $46.6 \pm 13.7(0.86 \pm 0.23 \mathrm{mg} / \mathrm{kg} /$ day $)$ prednisolone. Additionally, Rituximab \& IVIg were used in 2 patients each respectively. 3 months after initiation of immunosuppression 26/50 (52\%) patients on follow-up were in remission and $39 / 47(82.9 \%)$ patients attained remission at 6 months. Mean time to achieve remission was $151.1 \pm 86.3$ days. All IS agents were equally effective in inducing remission at 3 and 6 months and showed statistically similar BVAS reduction at 3 and 6 months from baseline (t test \& chi-square test). 7 (11.2\%) deaths noted in the cohort at their respective last visit during 195.3 patient years cumulative follow up. Multiple regression analysis showed that at baseline, presence of PNS involvement, eosinophilia, thrombocytosis, higher BVAS score and higher steroid requirement were predictors of persistently active vasculitis and absence of eye involvement and higher hemoglobin \% at baseline were predictors for remission, at 3 months $(p<0.05) .4 / 50(8 \%)$ patients had relapse of vasculitic symptoms. 2 and 5 year survival rates were $96.2 \%$ and $83.9 \%$ respectively Conclusions: Our cohort of Asian Indian RV was comparitively younger with lesser RA duration, less percentage of ever-smokers, lesser rheumatoid nodule prevalence, higher PNS involvement with better survival/mortality rates compared to published literature. All IS agents showed equal rates of BVAS remission \& BVAS reduction at 3 and 6 months of treatment

\section{References:}

[1] Scott DG, Bacon PA. Intravenous cyclophosphamide plus methylprednisolone in treatment of systemic rheumatoid vasculitis. Am J Med. 1984 Mar;76(3):37784.

[2] Mukhtyar C, Lee R, Brown D, Carruthers D, Dasgupta B, Dubey S, et al. Modification and validation of the Birmingham Vasculitis Activity Score (version 3). Ann Rheum Dis. 2009 Dec;68(12):1827-32.

Disclosure of Interest: None declared

DOI: 10.1136/annrheumdis-2017-eular.5993

\section{THU0323 THE INCIDENCE RATES OF ANCA-ASSOCIATED VASCULITIDES IN NORTHERN GERMANY (SCHLESWIG-HOLSTEIN) REMAIN STABLE BETWEEN 1998 TO 2014}

K. Herlyn ${ }^{1}$, W.L. Gross ${ }^{2}$, E. Reinhold-Keller ${ }^{2} \cdot{ }^{1}$ Rheumatology, Private Practice, Lübeck; ${ }^{2}$ Rheumatology, Klinikum Bad Bramstedt, Bad Bramstedt, Germany

Objectives: To assess the incidence of ANCA-associated vasculitides (AAV) in a stable population by registering all newly diagnosed patients in a large region in Northern Germany. To investigate the epidemiology and pattern of AAV over seventeen years in a prospective study.

Methods: Between 1/1/98 and 31/12/14 all newly diagnosed cases of AAV (Granulomatosis with polyangiitis (GPA), microscopic polyangitis (MPA), eosinophilic granulomatosis with polyangitis (EGPA)) as defined by the Chapel Hill Consensus Conference 1992 were identified in a large mixed rural/urban northern German region consisting of 2,83 million habitants in a population-based prospective study. The following sources were explored on a regular basis: (a) departments of all hospitals, including their outpatient clinics; (b) all departments of pathology, and (c) the reference immunological laboratories serving the catchment area. All cases were re-evaluated by the authors.

Results: Within the observation period of seventeen years 551 patients were identified as having a new diagnosis of AAV in the catchment area, the incidence rate was 12 (range $8-16) /$ million/year. GPA represents $70 \%$ of the AAV with an incidence rate of 7.9 (range $6-12) /$ million/year $(95 \% \mathrm{Cl} 1 ; 11$ - 5;19), MPA with an incidence rate of 2.4 (range 1-5)/million/year ( $\mathrm{Cl} 0 ; 3-1 ; 9)$ and EGPA 1.1 (range $0-2)$ /million/year (Cl 0;4).

Incidence rates of GPA were on average three times higher than those of MPA and seven times higher than CSS. The incidence rates were stable between 1998 and 2014 , our observations did not reveal significant differences. Gender differences were not observed. People diagnosed with MPA were on average 66.4 years old. Conclusions: Results of our unique population based vasculitis register over seventeen years assessing the incidence of AAV in a region of 2.83 million habitants in northern Germany demonstrate stable incidence rates for AAV. Variances between years have been observed, but no pattern or periodicity. Compared to other studies investigating small regions or referral centers the incidence rates for AAV were comparable to the incidence rates observed in Norway, Sweden and the United States, lower than those in United Kingdom, but higher than in Spain and Vilnius. The incidence rates of MPA are significantly higher in Japan, whereas GPA and CSS have rarely been diagnosed.

Disclosure of Interest: None declared

DOI: 10.1136/annrheumdis-2017-eular.3283

\section{THU0324 NEUTROPHILS IN GIANT CELL ARTERITIS: MONITORING DISEASE PROGRESSION DURING THERAPY TAPERING}

T. Kuret ${ }^{1}$, K. Lakota ${ }^{1,2}$, P. Žigon ${ }^{1}$, M. Ogrič ${ }^{1}$, Ž. Rotar $^{1}$, R. Ješe ${ }^{1}$ S. Sodin-Šemrl ${ }^{1,2}$, S. Čučnik ${ }^{1,3}, M$. Tomšič ${ }^{1,4}$, A. Hočevar ${ }^{1} .{ }^{1}$ Department of Rheumatology, University Medical Centre Ljubljana, Ljubljana; ${ }^{2}$ FAMNIT, University of Primorska, Koper; ${ }^{3}$ Faculty of Pharmacy; ${ }^{4}$ Faculty of Medicine, University of Ljubljana, Ljubljana, Slovenia

Background: Giant Cell Arteritis (GCA) represents a medical emergency due to risk of permanent vision loss and/or cerebrovascular insults. Polymyalgia rheumatic (PMR) frequently coexists with GCA ${ }^{1}$. No diagnostic or prognostic markers are yet known for GCA and predicting relapses during steroid therapy tapering is difficult. Biomarkers, such as serum amyloid A (SAA) and interleukin-6 (IL-6) provide added value for monitoring inflammation and a recent investigational study indicated the potential use of neutrophils and their surface markers in GCA pathogenesis ${ }^{2}$.

Objectives: To determine the percentage of neutrophils, $T$ and $B$ cells, and the mean fluorescence intensity (MFI) of L-selectin (CD62L) and integrin $\alpha M$ (CD11b) on $\mathrm{CD}_{16} 6^{+}$neutrophils in peripheral blood of newly diagnosed, untreated GCA and PMR patients vs. healthy controls (time 0 ) and at GCA follow-up (7,30 and 90 days after therapy).

Methods: Flow cytometry of stained, lysed and fixed whole blood ${ }^{2}$ was performed in 10 GCA patients ( 6 followed longitudinally), 7 PMR patients and 5 healthy controls (7-colour immunophenotyping kit, Miltenyi). Levels of SAA and IL-6 were measured in sera of GCA patients $(n=6)$ using nephelometry and ELISA, respectively.

Results: Percentage of neutrophils was significantly higher at time 0 in GCA and PMR patients compared to healthy controls. Expression of both CD62L and $\mathrm{CD} 11 \mathrm{~b}$ on $\mathrm{CD}_{16} 6^{+}$neutrophils was also higher in GCA and PMR patients, as compared to healthy controls. Longitudinally, GCA patients showed an initial decrease in percentage of neutrophils at day 7 in comparison with time 0 , increasing on days 30 and 90 , while both $\mathrm{T}\left(\mathrm{CD4}^{+}\right)$and $\mathrm{B}$ cells exhibited a significant elevation in $\%$ at day 7 , with a decline at days 30 and 90 . The MFI of neutrophil CD62L steadily decreased from day $0(85.80 \pm 45.45)$ to day 7 $(71.99 \pm 29.93)$ and day $30(57.61 \pm 42.17)$, while showing a substantial increase on day $90(88.54 \pm 60.35)$. CD11b expression diminished initially and remained reduced on day 90 . Levels of SAA and IL-6 declined sharply from day 0 to 7 (>10-fold drop) and day 30, with gradual elevation of both on day 90 .

Conclusions: Neutrophil CD62L may represent a good surface marker for monitoring disease progression following glucocorticoid tapering. SAA and IL-6 exhibit a sharp decrease at early time points, increasing at day 90 . In the future, a larger, longer study of neutrophils and their CD62L expression could support clinicians in their decision when and how to re-evaluate therapy in GCA patients. References:

[1] Schmidt J, Warrington KJ. Polymyalgia rheumatica and giant cell arteritis in older patients: diagnosis and pharmacological management. Drugs \& aging. 2011;28651-66.

[2] Nadkarni $S$ et al. Investigational analysis reveals a potential role for neutrophils in GCA disease progression. Circ.Res. 2014;114:242-48. 